\title{
Noninvasive Characterisation of Short- and Long-Term Recurrence of Atrial Signals During Persistent Atrial Fibrillation
}

\author{
Pietro Bonizzi ${ }^{1}$, Stef Zeemering ${ }^{2,3}$, Joël Karel ${ }^{1}$, Muhammad Haziq bin Kamarul Azman ${ }^{4}$, Theo \\ Lankveld $^{2,5}$, Ulrich Schotten ${ }^{2,3}$, Harry Crijns ${ }^{3,5}$, Ralf Peeters ${ }^{1}$, Olivier Meste $^{4}$ \\ ${ }^{1}$ Department of Data Science and Knowledge Engineering, Maastricht University, The Netherlands \\ ${ }^{2}$ Department of Physiology, Maastricht University Medical Center, The Netherlands \\ ${ }^{3}$ Cardiovascular Research Institute Maastricht (CARIM), Maastricht University, The Netherlands \\ ${ }^{4}$ Université Côte d'Azur, CNRS, I3S, France \\ ${ }^{5}$ Department of Cardiology, Maastricht University Medical Center, The Netherlands
}

\begin{abstract}
Propagation of electrical atrial activity $(A A)$ during atrial fibrillation $(A F)$ is a process characterized by different short- and long-term recurrence behaviours. Two antithetical (not mutually exclusive) hypotheses were proposed to noninvasively describe this nonstationary behaviour. The first hypothesis (H1) assumes a process with stationary spatial properties of AA propagation, but time-varying frequency properties, and vice versa for the second (H2). Based on $\mathrm{Hl}$ and $\mathrm{H} 2$, two phenomenological models were proposed, both able to replicate observations on AF patients, and a novel measure was introduced to assess the spatial variability of AA propagation (SVAAP) over short and long AA segments. Validity of the models was tested by looking at the relation between SVAAP-short and SVAAPlong on real observations from $A F$ patients (high-density body surface potential maps recorded in 75 patients affected by persistent AF). HI is confirmed if SVAAP-short is approximately equal to SVAAP-long. H2 if SVAAP-short is less than SVAAP-long. Results confirmed H2, showing that $A A$ propagation during $A F$ has strong nonstationary spatial properties. This could suggest new parameters to characterise AF substrate and predict therapy outcome.
\end{abstract}

\section{Introduction}

Propagation of electrical atrial activity (AA) during atrial fibrillation (AF) is a process characterised by different short- and long-term recurrence behaviours [1]. A detailed description of those differences may help to better characterise the AF substrate heterogeneity and complexity and suggest novel parameters to improve diagnosis and prediction of treatment outcome. This requires understanding the origin of these nonstationary behaviours. Although it is well known that AF is characterised by time- varying frequency properties [2,3], it is unclear whether this is the main contribution to the nonstationarity behaviour in the propagation of the $\mathrm{AF}$ wavefronts.

In this study, two antithetical (not mutually exclusive) hypotheses are suggested to noninvasively characterise the nonstationary behaviour of AA propagation during AF. The first hypothesis (H1) assumes a process with stationary spatial properties of AA propagation, but time-varying frequency properties. The second (H2) assumes a process with time-varying spatial properties and stationary frequency properties. Two models are implemented to reproduce each hypothesis, and a novel measure is suggested to quantify the amount of spatial variability in AA propagation. This measure is computed both on the output of the two models and on real observations from patients affected by persistent $\mathrm{AF}$, to test each hypothesis and select the one most in agreement with real data.

\section{Methods}

\subsection{BSPM data and pre-processing}

Body surface potential maps (BSPMs) were recorded in 75 patients in persistent $\mathrm{AF}$, with 120 anterior and 64 posterior leads (ActiveTwo BSM Panels Carbon Electrodes, Biosemi B.V., The Netherlands; Fig. 1). ECGs were sampled at $2048 \mathrm{~Hz}$, and downsampled at $256 \mathrm{~Hz}$. A oneminute segment was selected for each subject, low-quality leads were excluded (low signal-to-noise ratio, poor electrode contact, motion artefacts), and Wilson's Central Terminal was subtracted in line with conventional ECG analysis. After band-pass filtering the signals between 1 and $100 \mathrm{~Hz}$ (3rd order Chebyshev), QRST cancellation was performed using an adaptive singular value decomposition method, inspired by the approach in [4], with multiple QRST window templates defined using hierarchical clustering. The extracted AA signals were post-filtered with 


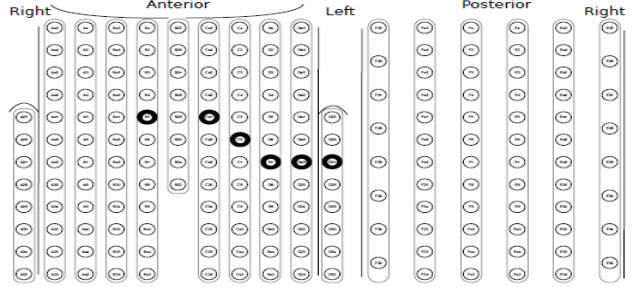

Figure 1. BSPM electrode configuration, comprising 120 anterior leads and 64 posterior leads. Thick circles: default positions of the precordial leads $V_{1}, \ldots, V_{6}$.

a zerophase notch filter at $50 \mathrm{~Hz}$ to suppress power line noise, and with a $3 \mathrm{~Hz}$ zero-phase highpass filter (3rd order Chebyshev) to remove low-frequency residuals not related to (persistent) AF.

\subsection{Multi-Variable AA Recurrence Signals}

Recently, we introduced a novel approach to describe the oscillatory patterns of AA signals during AF and well represent their recurrent behaviour over time, at the same time accounting for different short- and long-term recurrence behaviours [1]. The approach consists of generating an autocorrelation-like signal as follows: given the matrix $X$ of size $\ell \times N$ collecting all extracted AA signals from a patient $(\ell=184$ leads, and $N=60 \mathrm{~s} \cdot 256 \mathrm{~Hz}=15360$ samples), each column of $X$ is assumed to provide an $\ell \times 1$ vector $\mathbf{x}(n), n=1, \ldots, N$, which represents the overall spatial AA from all electrodes (hence multi-variable) at a given time instant. Then, a square matrix $R$ of size $M \times M$ is generated by computing:

$R_{i, j}=\frac{\mathbf{x}(i)^{T} \mathbf{x}(k)}{\|\mathbf{x}(i)\|_{2}\|\mathbf{x}(k)\|_{2}}, \begin{aligned} \text { with } i & =1, \ldots, M, \\ j & =1, \ldots, M, \\ \text { and } k & =i+j-1 .\end{aligned}$

Each entry of $R$ is therefore a measure of the cosine of the angle between two vectors, and provides a sort of normalized sample correlation. Moreover, column $j$ includes correlation values at lag $j-1$. The average over each column (per lag) is then computed, thus providing a multivariable autocorrelation function of the spatial AA oscillatory patterns, for lags $p=0, \ldots, M-1$. This function allows capture the recurrent behaviour of global AF spatial patterns over the body surface covered by the BSPM electrodes. In particular, assuming the AF cardiac dipole changes over time, this should affect the distance defined in (1). For instance, under the hypothesis that the AF dipole passes through the same points after a period $T$, the cosine of the angle between any two vectors which are $T$ seconds apart is expected to equal 1 . This procedure is repeated over nonoverlapping subsets of columns of $X$, called blocks, each block $m$ including $2 M$ columns.
A matrix $R^{(m)}$ and the corresponding autocorrelation signal can then be computed for each block $m$, to account for nonstationary behaviours in AF propagation. Those are defined as the multi-variable AA recurrence signals $r(p)^{(m)}$ $\left(p=0, \ldots, M-1 ; m=1, \ldots,\left\lfloor\frac{N}{2 M}\right\rfloor\right)$ for a patient. A value of $M=500$ was used in this study. This guaranteed to span approximately $4 \mathrm{~s}$ in each block, thus capturing several periods of AF. An example of multi-variable AA recurrence signals $r(p)^{(m)}$, computed over three consecutive blocks, is given in Fig. 2. Notice the variability in the shape of $r(p)^{(m)}$ from block to block, underlying the nonstationary behaviour of AF propagation patterns.

\subsection{Phenomenological models of AA prop- agation during AF}

Based on $\mathrm{H} 1$ and $\mathrm{H} 2$, two phenomenological models were generated, both able to generate pseudo-AA signals that can replicate the $r(p)^{(m)}$ signals computed on patients' data. The first model (M1) assumes a process with stationary spatial properties of AA propagation, but timevarying frequency properties, and vice versa for the second model (M2). The two models were generated from Eq. (2):

$$
\begin{array}{r}
\mathrm{M}_{k}(n)=\cos \left(2 \pi \frac{f(n)}{F_{s}} n+a \cdot s_{k}(n, d, v)+k / 2\right), \\
\text { with } f(n)=f_{A F}+b \cdot s(n, d, v), \\
\text { and } s(n, d, v) \text { being a random walk process. }
\end{array}
$$

$f_{A F}$ is the AF dominant frequency, and $k=1,2,3$ allows to generate 3-D loops which simulate the AA cardiac dipole during $\mathrm{AF}$ (also in case no randomness is introduced). M1 is obtained by setting $a=0$ and $b=1$, while $\mathrm{M} 2$ is obtained by setting $a=1$ and $b=0 . s(n, d, v)$ is a random walk process where each point is randomly drawn from the standard normal distribution, such that:

$$
\begin{array}{r}
s(n)=s(n-1)+\Delta, \\
\text { with } \Delta=v \cdot x, x \sim N(0,1) \text {, s.t. }|s(n)| \leq d .
\end{array}
$$

Hence, $d$ controls the range of $s(n)$ (the larger $d$ the larger the range), while $v$ controls the rate of variation of the increment (the larger $v$ the larger the rate of variation). The use of a random walk approach is suggested by what can be observed on real AA signals during AF. Indeed, for the same set of parameters $(d, v)$ (same AF substrate), it gives different dynamics w.r.t. where or when it is observed (as for the different dynamics of AF propagation captured by $r(p)^{(m)}$ over consecutive blocks). Dower's transform was finally used to map the three $\mathrm{M}_{k}(n)$ signals in (2) into a 12-D space to simulate 12-lead ECGs [5]. Fig. 3 shows examples of outputs of models M1 and M2. 

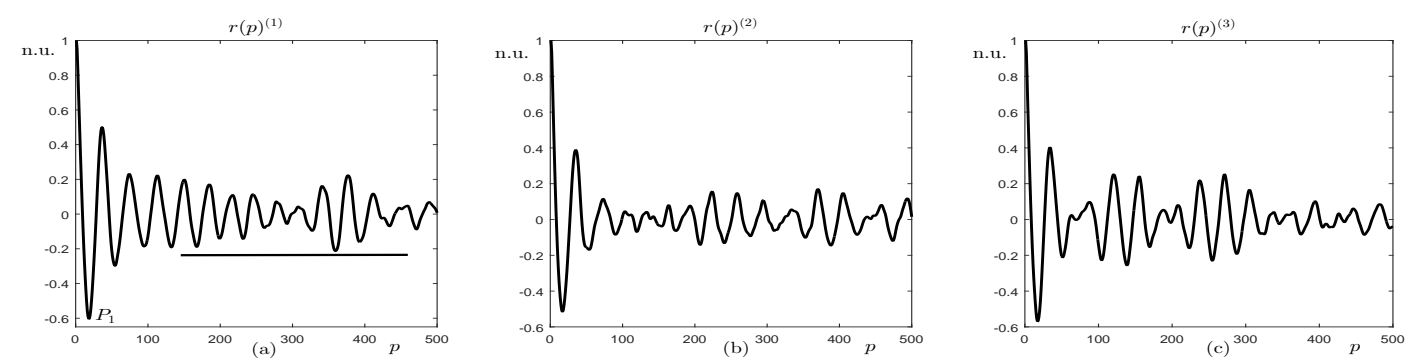

Figure 2. $r(p)^{(m)}$ curves from three consecutive blocks in a patient ( $m=1,2,3$ and $\left.p=0, \ldots, 499\right)$; n.u. is normalized units. In (a), the segment shows the constant portion of $r(p)^{(m)}$ whose mean absolute value is used as a measure of longterm recurrence behaviour of AA propagation (Section 2.5.3). The first negative peak $P_{1}$ in the $r(p)^{(m)}$ curve is also shown, which is used as a measure of short-term recurrence behaviour of AA propagation (Section 2.5.4).
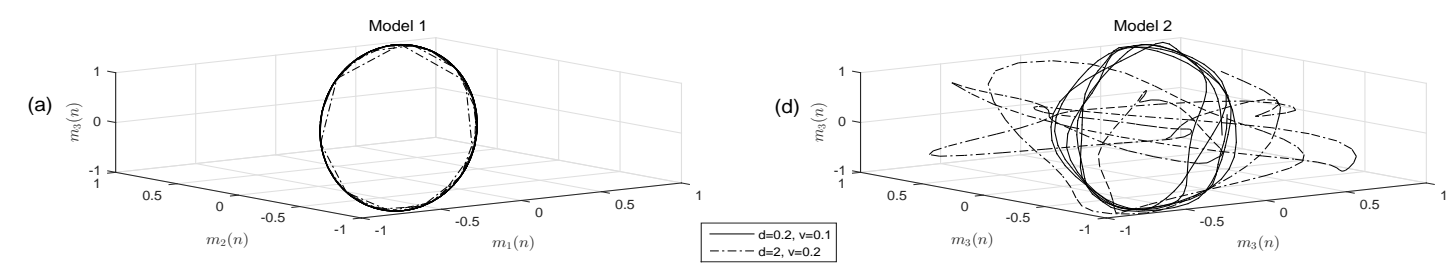

(b)

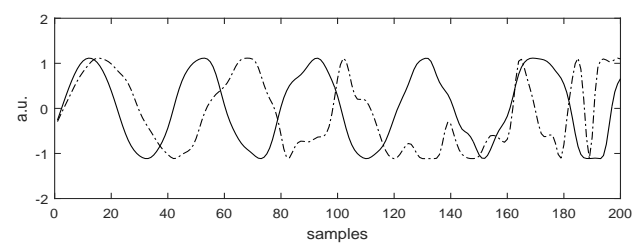

(c)

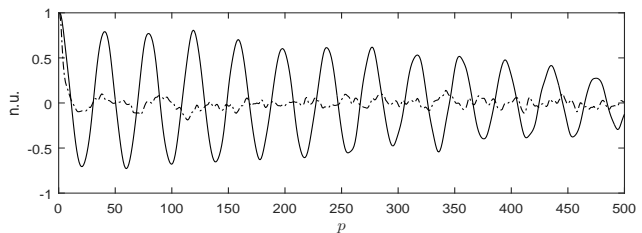

(e)

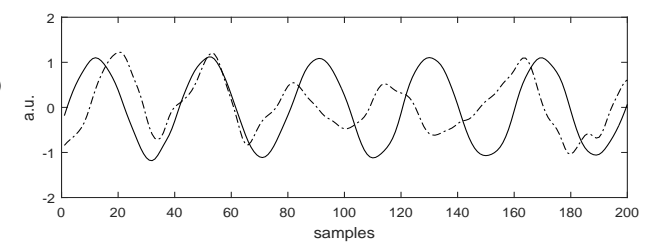

(f)

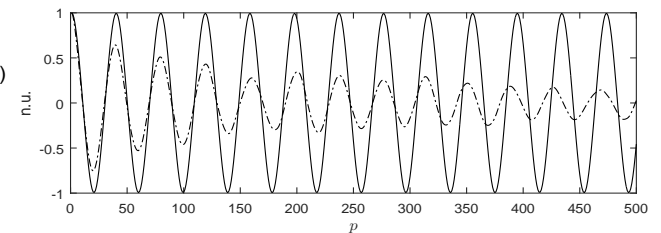

Figure 3. Examples of simulated 3-D cardiac dipoles ((a) and (d)), and corresponding pseudo-AA signals ((b) and (e)) and $r(p)^{(m)}$ curves ((c) and (f)), for model M1 (left column) and M2 (right column). Continuous lines corresponds to $s(n, 0.2,0.1)$, while dot-dashed lines to $s(n, 2,0.2)$. a.u. stands for arbitrary units. Only the first 200 samples are displayed in (a), (b), (d), and (e).

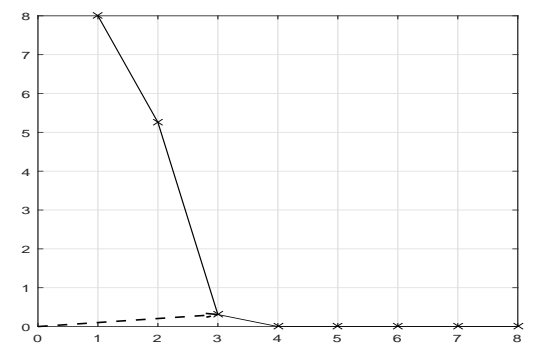

Figure 4. Example of calculation of SVAAP from the spectrum of a matrix. Indicated in dashed line is the point that minimises the distance with the origin.

\subsection{AA subspace dimension}

Spatial variability of AA propagation (SVAAP) was used as a measure to assess validity of both models in representing AF propagation dynamics in patients. SVAAP was assessed by computing AA subspace dimension. This was defined as the point in the spectrum of a matrix $\tilde{X}$ that minimises the distance between the plot of the spectrum and the origin (where $\tilde{X}$ can stand for either matrix $X$ or a part of $X$ of size $\ell \times 40$; see Fig. 4). The spectrum of $\tilde{X}$ was generated by applying Singular Value Decomposition to $\tilde{X}$, and then by scaling all singular values by $\frac{\ell}{\sigma_{1}}$ (where $\sigma_{1}$ is the first singular value). Scaling insures to have same units on both axis of the spectrum, and accurately estimate SVAAP. SVAAP was assessed over short and long AA segments. The whole signals were used to compute SVAAP-long, while SVAAP-short was computed over nonoverlapping segments of 40 samples (corresponding to one period of an $\mathrm{AF}$ waveform of frequency equal to $6.4 \mathrm{~Hz}$; average $\mathrm{AF}$ dominant frequency for all patients: $6.6 \pm 0.7 \mathrm{~Hz}$ ), and then averaged over all segments. 


\subsection{Analyses and Results}

1. Assessment of spatial variability of $A A$ propagation on $M 1$ and M2. SVAAP-short and SVAAP-long were first assessed on pseudo-AA signals from both M1 and M2, generated by means of Eq. (2). Several pseudo-AA signals were generated for each model, by randomly selecting parameters $d$ and $v$ from the empirical range $[0.1,5]$. As expected, M1 gave SVAAP-short approximately equal to SVAAP-long (stationary spatial properties and timevarying frequency properties, which do not affect AA subspace dimension), while M2 gave SVAAP-short less than SVAAP-long (time-varying spatial properties and stationary frequency properties). This confirms that M1 mainly represents $\mathrm{H} 1$, while $\mathrm{M} 2$ represents $\mathrm{H} 2$.

2. Assessment of spatial variability of AA propagation on patients' data. SVAAP-short and SVAAP-long were then assessed on the patients' data, to confirm either $\mathrm{H} 1$ or $\mathrm{H} 2$. $\mathrm{H} 1$ is confirmed if SVAAP-short is approximately equal to SVAAP-long (as for M1). H2 if SVAAP-short is less than SVAAP-long (as for M2). Results showed that SVAAPshort was significantly less than SVAAP-long $\left(\mathrm{p}<10^{-4}\right.$, Wilcoxon signed-rank test). This confirms H2, showing that AA propagation during AF is mainly a spatial nonstationary process.

3. Correlation between long-term spatial variability and long-term recurrence of $A A$ propagation. A visual inspection of $r(p)^{(m)}$ curves in Fig. 2 shows that they are characterized in general by two distinct behaviours: an early phase with a decreasing autocorrelation value, and a later phase with an approximately constant behaviour, and very low autocorrelation values [1]. The mean absolute value of the constant portion of each $r(p)^{(m)}$ signal was computed $(150 \leq p \leq 450$; Fig. 2(a)), and the average over all blocks was taken as a measure of long-term recurrence behaviour of AA propagation in a patient. Correlation between long-term spatial variability and long-term recurrence of AA propagation was then computed. SVAAPlong was negatively correlated with long-term recurrence of AA propagation $\left(\mathrm{R}=-0.50, p<10^{-4}\right)$.

4. Correlation between short-term spatial variability and short-term recurrence of AA propagation. The absolute value of the first negative peak in an $r(p)^{(m)}$ curve $\left(P_{1}\right.$ in Fig. 2(a)) can be interpreted as a measure of short-term recurrence behaviour of AA propagation in a patient. This peak occurs at approximately half a period of the AA propagation waveform (and thus also relates to the AF dominant frequency). Correlation between short-term spatial variability and short-term recurrence of AA propagation was computed. SVAAP-short was negatively correlated with $\left|P_{1}\right|\left(\mathrm{R}=-0.82, p<10^{-4}\right)$.

\section{Conclusions}

This study proposed two antithetical phenomenological models for noninvasively characterising short- and long- term dynamics of AA propagation during AF. One model assumes a process with stationary spatial properties of AA propagation, but time-varying frequency properties, and vice versa for the second. Both models were able to replicate AA recurrence signals as observed on patients suffering from persistent AF. However, only the second model showed spatial variability properties similar to those observed on real data. This suggests that AA propagation during $\mathrm{AF}$ is not only characterised by time-varying frequency properties, but also and mainly by time-varying spatial properties. Moreover, the negative correlation between SVAAP-long and long-term recurrence of AA propagation suggests that the more organised AA is (and the less complex the AF substrate) the more stable in time and space its propagation is (fewer propagation paths), and vice versa. Additionally, the negative correlation between SVAAP-short and short-term recurrence of AA propagation suggests more stable loops (reentries) over short time windows. These results are in agreement with the fact that a more progressed AF (and a more complex AF substrate) manifests in more disorganised and nonstationary AA signals [6]. At the same time, this novel approach may be able to capture more details about AF propagation dynamics and be able to discriminate among patients characterised by very similar AF substrate complexity. This could suggest new parameters to predict therapy outcome.

\section{References}

[1] Meste O, Zeemering S, Karel J, Lankveld T, Schotten U, Crijns H, Peeters R, Bonizzi P. Noninvasive Recurrence Quantification Analysis Predicts Atrial Fibrillation Recurrence in Persistent Patients Undergoing Electrical Cardioversion. In Proc. Computing in Cardiology 2016; 43.

[2] Stridh M, Sörnmo L, Meurling CJ, Olsson SB. Characterization of atrial fibrillation using the surface ECG: timedependent spectral properties. IEEE Trans Biomed Eng 2001;48(1):19-27.

[3] Stridh M, Husser D, Bollmann A, Sörnmo L. Waveform characterization of atrial fibrillation using phase information. IEEE Trans Biomed Eng 2009;56:1081-1089.

[4] Alcaraz R, Rieta JJ. Adaptive singular value cancellation of ventricular activity in single-lead atrial fibrillation electrocardiograms. Physiol Meas 2008;29:1351-1369.

[5] Dower G, Machado H, Osborne J. On deriving the electrocardiogram from vectoradiographic leads. Clin Cardiol 1980; 3(2):87-95.

[6] Lankveld TAR, Zeemering S, Crijns HJGM, Schotten U. The ECG as a tool to determine atrial fibrillation complexity. Heart 2014;100:1077-84.

Address for correspondence:

Pietro Bonizzi

P.O. Box 616, 6200 MD, Maastricht, The Netherlands

pietro.bonizzi@maastrichtuniversity.nl 